S. KIMURA

KODAI MATH. J.

9 (1986). $351-360$

\title{
A CHARACTERIZATION OF THE EXPONENTIAL FUNCTION AND LINDELÖF FUNCTION
}

\author{
By SHIGERU KimURA
}

$\S 1$. In our previous papers $[1,2]$, we considered the entire functions which have only negative zeros and obtained a characterization of the exponential function. In this paper we consider the entire functions whose zeros are distributed in a sector and prove the followings.

THEOREM 1. Suppose that $f(z)$ is an entire function of order $q=2 p+1$ where $p$ is non-negative integer and having zeros $\left\{a_{\nu}\right\}$ in the sector $\{z ; 0 \leqq \arg z-\pi \leqq$ $\beta<\pi /(q+1)\}$ and $\Sigma\left|a_{\nu}\right|^{-q}<+\infty$. Let $\phi\left(z^{2}\right)=f(z) f(-z)$ and $g(z)=\phi(-z) / \phi(0)$ Unless $g(z) \equiv 1$, we assume that $g(z)$ is a canonical product and

$$
(-1)^{k} \log \left|g\left(r e^{i \beta}\right)\right| \leqq(-1)^{k}(\cos \beta q / 2) \log |g(r)|+\varepsilon(r)(-1)^{k} \log |g(r)|
$$

for all sufficiently large $r$ where $k$ is the genus of $g(z), 0<\beta<\pi /(q+1)$ and $0 \leqq \varepsilon(r)=0\left(1 / r^{\varepsilon_{0}}\right), \quad \varepsilon_{0}>0$. Then $f(z)=e^{P(z)}$ where $P(z)$ is an odd polynomial of degree $q$.

As an improvement of Theorem 1 we have

THEOREM 1'. Suppose that $f(z)$ is an entire function of order $q=2 p+1$ where $p$ is non-negative integer and having zeros $\left\{a_{\nu}\right\}$ in the sector $\{z ; 0 \leqq \arg z-\pi$ $\leqq \beta<\pi /(q+1)\}$ and $\Sigma\left|a_{\nu}\right|^{-q}<+\infty$. Let $\phi\left(z^{2}\right)=f(z) f(-z), \quad g(z)=\phi(-z) / \phi(0)=$ $e^{Q(z)} g_{0}(z)$ and $h(z)=e^{Q(z)} \overline{g_{0}(\bar{z})}$ where $g_{0}(z)$ is a canonical product. We assume that

$$
(-1)^{k} \log |g(r)| \geqq 0
$$

and

$$
\begin{aligned}
(-1)^{k} \log \left|g\left(r e^{i \beta}\right) h\left(r e^{-i \beta}\right)\right| \leqq & 2(-1)^{k}(\cos \beta q / 2) \log |g(r)| \\
& +\varepsilon(r)(-1)^{k} \log |g(r)|
\end{aligned}
$$

for all sufficiently large $r$ where $k$ is the genus of $g_{0}(z), 0<\beta<\pi /(q+1)$ and $0 \leqq \varepsilon(r)=0\left(1 / r^{\varepsilon_{0}}\right), \varepsilon_{0}>0$. Then $f(z)=e^{P(z)}$ where $P(z)$ is a polynomial of degree $q$.

Omitting the condition about zeros $\left\{a_{\nu}\right\}$, we prove the following theorems.

Received October 9, 1985 
THEOREM 2. Suppose that $f(z)$ is an entire function of order one and having zeros in the sector $\{z ;|\arg z-\pi| \leqq \beta<\pi / 4\}$ such that

$$
f(r) f(-r)=O(1) \quad(r \rightarrow \infty) .
$$

Then $f(z)=A e^{B z}$, where $A, B$ are constants, or else

$$
\lim _{r \rightarrow \infty} \frac{\log M(r, f)}{r}=\infty .
$$

THEOREM 3. Suppose that $f(z)$ is an entire function of order $q=2 p+1$ where $p$ is non-negative integer and having zeros in the sector $\{z ; 0 \leqq \arg z-\pi \leqq$ $\beta<\pi / 2(q+1)\}$. Setting $\phi\left(z^{2}\right)=f(z) f(-z), g(z)=\phi(-z) / \phi(0)$, we assume that $g(z)$ is a canonical product and

$$
(-1)^{k} \log \left|g\left(r e^{i \beta}\right)\right| \leqq(-1)^{k}(\cos \beta q / 2) \log |g(r)|
$$

for all sufficiently large $r$ where $k$ is the genus of $g(z)$ and $0<\beta<\pi / 2(q+1)$. Then $f(z)=e^{P(z)}$ where $P(z)$ is an odd polynomial of degree $q$, or else

$$
\lim _{r \rightarrow \infty} \frac{\log M(r, f)}{r^{q}}=\infty .
$$

These two theorems are the extensions of those in [1] whose functions have only negative zeros.

As an improvement of Theorem 3 we have

THEOREM 3'. Suppose that $f(z)$ is an entire function of order $q=2 p+1$ where $p$ is non-negative integer and having zeros in the sector $\{z ; 0 \leqq \arg z-\pi \leqq$ $\beta<\pi / 2(q+1)\}$. Setting $\phi\left(z^{2}\right)=f(z) f(-z), g(z)=\phi(-z) / \phi(0)=e^{Q(z)} g_{0}(z)$ and $h(z)=$ $e^{Q(z)} \overline{g_{0}(\bar{z})}$ where $g_{0}(z)$ is a canonical product, we assume that

and

$$
(-1)^{k} \log |g(r)| \geqq 0
$$

$$
(-1)^{k} \log \left|g\left(r e^{i \beta}\right) h\left(r e^{-\imath \beta}\right)\right| \leqq 2(-1)^{k}(\cos \beta q / 2) \log |g(r)|
$$

for all sufficiently large $r$ where $k$ is the genus of $g_{0}(z)$ and $0<\beta<\pi / 2(q+1)$. Then $f(z)=e^{P(z)}$ where $P(z)$ is a polynomial of degree $q$, or else

$$
\lim _{r \rightarrow \infty} \frac{\log M(r, f)}{r^{q}}=\infty .
$$

As a characterization of the entire function and Lindelöf function we prove the following theorems.

THEOREM 4. Suppose that $f(z)$ is an entire function of order one and having zeros in the sector $\{z ;|\arg z-\pi| \leqq \beta<\pi / 4\}$ such that 


$$
f(r) f(-r)=O(1) \quad(r \rightarrow \infty)
$$

and

$$
\liminf _{r \rightarrow \infty} \frac{\log |f(i r) f(-i r)|}{r}<\infty .
$$

Then $f(z)=A e^{B z}$, or else $n(r, f) \sim \alpha r$ where $n(r, f)$ indicates the number of zeros of $f(z)$ in $|z|<r$ and $\alpha$ is a constant.

THEOREM 5. Suppose that $f(z)$ is an entire function of order $q=2 p+1$ where $p$ is non-negative integer and having zeros in the sector $\{z ; 0 \leqq \arg z-\pi \leqq \pi / 2(q+1)\}$. Setting $\phi\left(z^{2}\right)=f(z) f(-z), g(z)=\phi(-z) / \phi(0)$, we assume that $g(z)$ is a canonical product and

$$
(-1)^{k} \log \left|g\left(r e^{i \beta}\right) \leqq(-1)^{k}(\cos \beta q / 2) \log \right| g(r) \mid
$$

for all sufficiently large $r$ where $k$ is the genus of $g(z)$ and $0<\beta<\pi / 2(q+1)$. Further we assume that

$$
\liminf _{r \rightarrow \infty} \frac{|\log | f(i r) f(-\imath r)||}{r}<\infty .
$$

Then $f(z)=e^{P(z)}$ where $P(z)$ is an odd polynomial of degree $q$, or else $n(r, f) \sim \alpha r^{q}$ where $n(r, f)$ indicates the number of zeros of $f(z)$ in $|z|<r$ and $\alpha$ is a constant.

As an improvement of Theorem 5 we have

THEOREM 5'. Suppose that $f(z)$ is an entire function of order $q=2 p+1$ where $p$ is non-negative integer and having zeros in the sector $\{z ; 0 \leqq \arg z-\pi \leqq \pi / 2(q+1)\}$. Setting $\phi\left(z^{2}\right)=f(z) f(-z), g(z)=\phi(-z) / \phi(0)=e^{Q(z)} g_{0}(z)$ and $h(z)=e^{Q(z)} \overline{g_{0}(\bar{z})}$ where $g_{0}(z)$ is a canonical product, we assume that

and

$$
(-1)^{k} \log |g(r)| \geqq 0
$$

$$
(-1)^{k} \log \left|g\left(r e^{i \beta}\right) h\left(r e^{-i \beta}\right)\right| \leqq(-1)^{k} 2(\cos \beta q / 2) \log |g(r)|
$$

for all sufficiently large $r$ where $k$ is the genus of $g(z)$ and $0<\beta<\pi / 2(q+1)$. Further we assume that

$$
\liminf _{r \rightarrow \infty} \frac{|\log | f(i r) f(-i r)||}{r^{q}}<\infty .
$$

Then $f(z)=e^{P(z)}$ where $P(z)$ is a polynomial of degree $q$, or else $n(r, f) \sim \alpha r^{q}$ where $n(r, f)$ indicates the number of zeros of $f(z)$ in $|z|<r$ and $\alpha$ is a constant.

The proofs of our theorems depends on those of theorems in [1] and the following lemma. 
LEMMA 1 [3]. Let

$$
\phi(x, y)=(1 / 2) \log \left(1+2 y \cos x+y^{2}\right)+\sum_{j=1}^{k}(-1)^{\jmath}\left(y^{\jmath} / j\right) \cos j x .
$$

Then $(-1)^{k} \psi(x, y)$ is monotone decreasing for $0 \leqq x \leqq \pi /(k+1), y \geqq 0$.

§2. Proof of Theorem 1 and Theorem $\mathbf{1}^{\prime}$. Let $f(z)$ be an entire function satisfying the hypotheses in Theorem 1 . We can write

$$
f(z)=e^{P(z)} \prod_{\nu=1}^{\infty} E\left(z / a_{\nu}, q-1\right)
$$

where $P(z)$ is a polynomial of degree at most $q$ and $E$ is the Weierstrass primary factor. Defining

$$
f^{*}(z)=e^{P(z)} \prod_{\nu=1}^{\infty} E\left(-z /\left|a_{\nu}\right|, q-1\right)
$$

and $\phi^{*}\left(z^{2}\right)=f^{*}(z) f^{*}(-z)$, we have

$$
\log M\left(r^{2}, \phi^{*}\right)=o\left(r^{q}\right) .
$$

Hence $g^{*}(z)=\phi^{*}(-z) / \phi(0)$ satisfies

$$
\lim _{r \rightarrow \infty} \frac{\log M\left(r, g^{*}\right)}{r^{q / 2}}=0 .
$$

If $g(z) \equiv 1$, then $f(z)=e^{P(z)}$ where $P(z)$ is a polynomial of degree $q$, which is the desired result.

Now we assume that $g(z)$ is a canonical product of genus $k$. Since

$$
\phi\left(x, r /\left|b_{\nu}\right|\right)=\log \left|E\left(-r e^{\imath x} /\left|b_{\nu}\right|, k\right)\right|,
$$

by the definition of $\phi$ in Lemma 1 , we have

$$
\sum_{\nu=1}^{\infty} \psi\left(x, r /\left|b_{\nu}\right|\right)=\log \left|g^{*}\left(r e^{\imath x}\right)\right|
$$

where $\left\{b_{\nu}\right\}$ are zeros of $g(z)$.

Since $\left\{b_{\nu}\right\}$ are distributed in the sector $\{z ; 0 \leqq \arg z-\pi \leqq 2 \beta<2 \pi /(q+1)\}$ and $k \leqq(q-1) / 2$, we can easily see that

$$
(-1)^{k} \log |g(r)| \leqq(-1)^{k} \log \left|g^{*}(r)\right|
$$

and

$$
(-1)^{k} \log \left|g^{*}\left(r e^{2 \beta}\right)\right| \leqq(-1)^{k} \log \left|g\left(r e^{i \beta}\right)\right|
$$

by Lemma 1 .

Thus we have from (1.1) 


$$
(-1)^{k} \log \left|g^{*}\left(r e^{i \beta}\right)\right| \leqq(-1)^{k}(\cos \beta q / 2) \log \left|g^{*}(r)\right|+\varepsilon(r)(-1)^{k} \log \left|g^{*}(r)\right| .
$$

From this inequality we can induce

$$
C_{1} \frac{|\log | g^{*}(r)||}{r^{q / 2}}-C_{2} \frac{\log M\left(2 s, g^{*}\right)}{(2 s)^{q / 2}} \leqq C \frac{|\log | g^{*}(r)||}{r^{(q / 2)+\varepsilon_{0}}}
$$

by proceeding as in $\S 4$ of [1], for a sequence of $r=\left\{r_{n}\right\}$ tending to infinity with $n$ where $C_{1}, C_{2}, C$ are positive constants which do not depend on $r$. Hence we arrive at an impossible inequality from (2.1) and $\varepsilon_{0}>0$. This completes the proof of Theorem 1 .

In order to prove Theorem $1^{\prime}$, firstly we consider the case $\operatorname{deg}(\operatorname{Re} Q(r))=0$ and $g_{0}(z)=1$. Then we deduce $f(z)=e^{P(z)}$ where $P(z)$ is a polynomial of degree $q$, which is the desired result.

Secondly we assume that (1.2) and (1.3) hold. Then we can prove the following inequality by Lemma 1 , the definition of $h(z)$ and (1.3),

$$
(-1)^{k} \log \left|g^{*}\left(r e^{i \beta}\right) g^{*}\left(r e^{-i \beta}\right)\right| \leqq 2(-1)^{k}(\cos \beta q / 2) \log \left|g^{*}(r)\right|+\varepsilon(r) \log \left|g^{*}(r)\right|,
$$

where $g^{*}(z)=e^{Q(z)} g_{0}^{*}(z)$. Therefore, proceeding as in the proof of Theorem 1 , we can prove Theorem 1'.

§ 3. Proof of Theorem 2. Let $f(z)$ be an entire function satisfying the hypotheses in Theorem 2. We suppose that (1.5) is false, i.e.,

$$
\liminf _{r \rightarrow \infty} \frac{\log M(r, f)}{r^{1 / 2}}<\infty .
$$

Let $\phi\left(z^{2}\right)=f(z) f(-z), g(z)=\phi(-z) / \phi(0)$. If $g(z)$ is constant, then we have $f(z)=A e^{B z}$ where $A, B$ are constants, which is the desired result. In order to complete the proof of Theorem 2, we may thus confine ourselves to the case when $g(z)$ is not constant. We shall show that this will contradict (3.1), so that (1.5) must hold as required.

By (3.1) and $\log M\left(r^{2}, \phi\right) \leqq 2 \log M(r, f)$, we have

$$
\liminf _{r \rightarrow \infty} \frac{\log |g(r)|}{r^{1 / 2}}<\infty .
$$

Since the genus of $g(z)$ is zero, we can write

$$
g(z)=\prod_{\nu=1}^{\infty}\left(1+z / b_{\nu}\right), \quad\left|\arg b_{\nu}\right| \leqq 2 \beta<\pi / 2 .
$$

Setting

$$
g^{*}(z)=\prod_{\nu=1}^{\infty}\left(1+z /\left|b_{\nu}\right|\right)
$$

we see that 


$$
\liminf _{r \rightarrow \infty} \frac{\log \left|g^{*}(r)\right|}{r^{1 / 2}}<\infty
$$

In fact, by Lemma 1

$$
\begin{aligned}
\log |g(r)| & \geqq \log \left|g^{*}\left(r e^{i 2 \beta}\right)\right|=r \int_{0}^{\infty} \frac{n(x)}{x} \frac{x \cos 2 \beta+r}{x^{2}+r^{2}+2 x r \cos 2 \beta} d x \\
& \geqq r \cos 2 \beta \int_{0}^{\infty} \frac{n(x)}{x} \frac{d x}{x+r}=(\cos 2 \beta) \log \left|g^{*}(r)\right| .
\end{aligned}
$$

Hence we have (3.3) in view of (3.2).

Since (1.4) is equivalent to $g(-r)=0(1)$ and $\left|g^{*}(-r)\right| \leqq|g(-r)|$ by Lemma 1 , we have $g^{*}(-r)=0(1)$. Now we may assume that

$$
\log \left|g^{*}(-r)\right| \leqq 0 \text {. }
$$

Arguing as in $\S 5$ of [1], we have

$$
\int_{r}^{s} \frac{\log \left|g^{*}(-t)\right|}{t^{1 / 2}} d t>C_{1} \frac{\log \left|g^{*}(r)\right|}{r^{1 / 2}}-C_{2} \frac{\log \left|g^{*}(2 s)\right|}{(2 s)^{1 / 2}} \quad(0<r<s<+\infty)
$$

where $C_{1}, C_{2}$ are positive constants which do not depend on $r$.

Case (1). $B=\limsup _{r \rightarrow \infty} \frac{\log \left|g^{*}(r)\right|}{r^{1 / 2}}=\infty$. From (3.3) we can find arbitrarily large values of $r$ and $s$, with $r<s$, such that the right-hand side of (3.5) is positive. Thus it follows that the inequality

$$
\log \left|g^{*}(-t)\right|>0
$$

holds for some $t>r$ and this contradicts (3.4).

Case (2). $B=0$. In any case, we have $(\log |g(r)|) / r^{1 / 2}>0$ for $r>0$. For each fixed $r$ the right-hand side of (3.5) is positive for sufficiently large $s$, and again we have a contradiction.

Case (3). $0<B<+\infty$. Arguing as in $\S 5$ of [1], we have

$$
\log \left|g^{*}(r)\right| \leqq \int_{0}^{\infty}\left(\log \left|g^{*}(t)\right|\right) Q(r, t) d t
$$

where $Q(r, t)=2 r \pi^{-2}\left(r^{2}-t^{2}\right)^{-1} \log r t^{-1}$. Thus, proceeding as in $\S 5$ of [1], we arrive at

$$
\lim _{r \rightarrow \infty} \frac{\log \left|g^{*}(r)\right|}{r^{1 / 2}}=B>0
$$

Hence, by Valiron's Tauberian Theorem [4], we have

$$
n\left(r, 0, g^{*}\right) \sim(B / \pi) r^{1 / 2}
$$

and

$$
n\left(r, 0, f^{*}\right) \sim(B / \pi) r
$$


where $f^{*}(z)$ is the entire function such that if $f(z)$ is represented as

$$
f(z)=A e^{B z} \prod_{\nu=1}^{\infty} E\left(-\frac{z}{a_{\nu}}, 1\right)=A e^{B z} f_{0}(z)
$$

then

$$
f^{*}(z)=A e^{B z} \prod_{\nu=1}^{\infty} E\left(-\frac{z}{\left|a_{\nu}\right|}, 1\right)=A e^{B z} f_{0}^{*}(z) .
$$

Therefore we have $\delta\left(0, f_{0}^{*}\right)=1$ and so

$$
\delta\left(0, f^{*}\right)=1
$$

Now we can show that

$$
\liminf _{r \rightarrow \infty} \frac{-\log \left|f^{*}(r)\right|}{r}<\infty .
$$

Let $z=r e^{i \theta}, a_{\nu}=\left|a_{\nu}\right| e^{\imath \phi \nu}, y_{\nu}=r /\left|a_{\nu}\right|$. Look at values of $\phi\left(\theta-\phi_{\nu}, y_{\nu}\right)$ in $0 \leqq \theta \leqq$ $\pi / 4-\beta$. By the assumption $\left|\phi_{\nu}\right| \leqq \beta$. Then by Lemma 1 ,

$$
\phi\left(\theta-\phi_{\nu}, y_{\nu}\right)=-\psi\left(-\theta+\phi_{\nu}, y_{\nu}\right) \geqq-\phi\left(\theta+\left|\phi_{\nu}\right|, y_{\nu}\right) \text {. }
$$

Hence we have

$$
-\log \left|f_{0}^{*}\left(r e^{i \theta}\right)\right| \geqq-\log \left|f_{0}^{*}\left(r e^{i(\theta+\beta)}\right)\right| .
$$

Let $\varepsilon$ a sufficiently small positive number such that $\varepsilon<\pi / 4-\beta$. Then for $0 \leqq \theta \leqq \pi / 4-\beta-\varepsilon$

$$
\begin{aligned}
-\log \left|f_{0}^{*}\left(r e^{2(\theta+\beta)}\right)\right| & =r^{2} \int_{0}^{\infty} \frac{n(x)}{x^{2}} \frac{x \cos 2(\theta+\beta)+r \cos (\theta+\beta)}{x^{2}+r^{2}+2 x r \cos (\theta+\beta)} d x \\
& \geqq-\cos \left(\frac{\pi}{2}-2 \varepsilon\right) \log \left|f_{0}^{*}(r)\right| .
\end{aligned}
$$

Thus, setting $\theta_{0}=\pi / 4-\beta-\varepsilon$, we have

$$
\begin{aligned}
& \int_{0}^{\theta_{0}} \log +\frac{1}{\left|f_{0}\left(r e^{i \theta}\right)\right|} d \theta=-\int_{0}^{\theta_{0}} \log \left|f_{0}\left(r e^{i \theta}\right)\right| d \theta \\
& \geqq-\int_{0}^{\theta_{0}} \log \left|f_{0}^{*}\left(r e^{i(\theta+\beta)}\right)\right| d \theta \geqq-\theta_{0}\left(\cos \left(\frac{\pi}{2}-2 \varepsilon\right)\right) \log \left|f_{0}^{*}(r)\right| .
\end{aligned}
$$

Therefore we have (3.7) from (3.1) and $\log M\left(r, f_{0}\right) \leqq \log M\left(r, e^{-B z}\right)+\log M(r, f)$. Proceeding as in $\S 4$ of [1] from (3.6) and (3.7), we have $B=0$, which is impossible.

$\S 4$. Proof of Theorem 3 and Theorem $3^{\prime}$. In order to prove Theorem 3 and Theorem $3^{\prime}$, we prove the following lemma.

LEMMA 2. Let $g(z)$ be a canonical product of genus $k$ and having zeros 
$\left\{-b_{\nu}\right\}$ in the sector $\{z ; 0 \leqq \arg z-\pi \leqq 2 \beta\}$ where $\beta$ is a positive number such that $2 \beta(k+1)<\pi / 2$. Let $g^{*}(z)$ be a canonical product of genus $k$ and having only negative zeros $\left\{-\left|b_{\nu}\right|\right\}$. Then we have

$$
(-1)^{k} \log |g(r)| \geqq(-1)^{k}(\cos 2 \beta(k+1)) \log \left|g^{*}(r)\right|
$$

and especially $(-1)^{k} \log |g(r)|>0$.

Let $z=r e^{\imath x}, y_{\nu}=r /\left|b_{\nu}\right|$. Then we have $\sum \psi\left(x, y_{\nu}\right)=\log \left|g^{*}\left(r e^{\imath x}\right)\right|$, and so by Lemma 1 we have

$$
\begin{aligned}
& (-1)^{k} \log |g(r)| \geqq(-1)^{k} \log \left|g^{*}\left(r e^{i 2 \beta}\right)\right| \\
& =(-1)^{2 k} r^{k+1} \int_{0}^{\infty} \frac{n(x)}{x^{k+1}} \frac{x \cos 2 \beta(k+1)+r \cos 2 \beta k}{x^{2}+r^{2}+2 r x \cos 2 \beta} d x \\
& \geqq(-1)^{k}(\cos 2 \beta(k+1)) \log \left|g^{*}(r)\right| .
\end{aligned}
$$

In order to prove Theorem 3 , let $f(z)$ be an entire function satisfying the hypotheses in Theorem 3 . We assume that (1.7) is false, i. e.,

$$
\liminf _{r \rightarrow \infty} \frac{\log M(r, f)}{r^{q}}<\infty .
$$

If $g(z)$ is constant, then we have $f(z)=e^{P(z)}$ where $P(z)$ is a polynomial of degree $q$, which is the desired result. Hence we may assume that $g(z)$ is not constant. Since $2 k \leqq q-1$ and $2 \beta(q+1)<\pi$, we have $2 \beta(k+1)<\pi / 2$. Therefore we can show that

$$
\liminf _{r \rightarrow \infty} \frac{(-1)^{k} \log \left|g^{*}(r)\right|}{r^{q / 2}}<\infty .
$$

If $k$ is even, then (4.1) and (4.2) imply (4.3). If $k$ is odd, then we have

$$
\int_{0}^{\theta_{0}} \log ^{+} \frac{1}{\left|g\left(r e^{i \theta}\right)\right|} d \theta \geqq-\theta_{0}\left(\cos \left(\frac{\pi}{2}-\varepsilon(k+1)\right)\right) \log \left|g^{*}(r)\right|
$$

where $\theta_{0}=\pi / 2(k+1)-2 \beta-\varepsilon$ for a sufficiently small positive $\varepsilon$ such that $\varepsilon<\pi / 2(k+1)-2 \beta$, and hence we have (4.3) from (4.2).

On the other hand we have from (1.6) and Lemma 1,

$$
(-1)^{k} \log \left|g^{*}\left(r e^{i \beta}\right)\right| \leqq(-1)^{k}(\cos \beta q / 2) \log \left|g^{*}(r)\right| .
$$

Arguing as in $\S 5$ of [1], we have

$$
\begin{aligned}
& \int_{r}^{s i} \frac{(-1)^{k} \log \left|g^{*}\left(t e^{i \beta}\right)\right|-(-1)^{k}(\cos \beta q / 2) \log \left|g^{*}(t)\right|}{t^{1+q / 2}} d t \\
& >C_{1} \frac{(-1)^{k} \log \left|g^{*}(r)\right|}{r^{q / 2}}-C_{2} \frac{(-1)^{k} \log \left|g^{*}(2 s)\right|}{(2 s)^{q / 2}} \quad(0<r<s<+\infty) .
\end{aligned}
$$


Let

$$
B=\limsup _{r \rightarrow \infty}\left((-1)^{k} \log \left|g^{*}(r)\right|\right) / r^{q / 2} .
$$

If $B$ is $+\infty$ or 0 , then we have a contradiction from (4.3), (4.4) and (4.5). If $0<B<+\infty$, then

$$
(-1)^{k} \log \left|g^{*}\left(r^{r}\right)\right| \leqq \int_{0}^{\infty}(1+\cos \beta q / 2)(-1)^{k}\left(\log \left|g^{*}\left(t^{r}\right)\right|\right) Q(r, t) d t
$$

where $\gamma=\beta / \pi$. Hence

$$
\lim _{r \rightarrow \infty} \frac{(-1)^{k} \log \left|g^{*}\left(r^{r}\right)\right|}{r^{\gamma q / 2}}=B .
$$

Therefore $\delta\left(0, f^{*}\right)=1$. Now we can show that

$$
\liminf _{r \rightarrow \infty} \frac{-\log \left|f^{*}(r)\right|}{r^{q}}<\infty .
$$

In fact, setting $\theta_{0}=\pi / 2(q+1)-\beta-\varepsilon$ for a sufficiently small positive $\varepsilon$ such that $\varepsilon<\pi / 2(q+1)-\beta$, we have

$$
\int_{0}^{\theta_{0}} \log ^{+} \frac{1}{\left|f_{0}\left(r e^{i \theta}\right)\right|} d \theta \geqq-\theta_{0}\left(\cos \left(\frac{\pi}{2}-\varepsilon(q+1)\right)\right) \log \left|f_{0}^{*}(r)\right|,
$$

where $f_{0}(z)$ is the canonical product formed with the zeros of $f(z)$. Hence we have (4.6) from (4.2). Proceeding as in $\S 4$ of [1], we have $B=0$, from $\delta\left(0, f^{*}\right)=1$ and (4.6), which is impossible.

In order to prove Theorem $3^{\prime}$, it is necessary to prove (4.3), the inequality corresponding to (4.4) and (4.6) in our case. The proof of (4.6) in our case is quite similar to that of (4.6) in Theorem 3. The proof of (4.3) in our case follows from the similar arguments to those in the proof of Theorem 3 , observing that $g^{*}(z)=e^{Q(z)} g_{0}^{*}(z)$ where $g_{0}^{*}(z)$ is the canonical product formed with the zeros of $g^{*}(z)$ and $Q(z)$ is a polynomial with $\operatorname{deg} Q(z) \leqq p$. Now by Lemma $1,(1.8)$ and the definition of $h(z)$, we have the following inequality corresponding to (4.4),

$$
(-1)^{k} \log \left|g^{*}\left(r e^{i \beta}\right) g^{*}\left(r e^{-i \beta}\right)\right| \leqq 2(-1)^{k}(\cos \beta q / 2) \log \left|g^{*}(r)\right| .
$$

Thus proceeding as in the proof of Theorem 3, we can prove Theorem $3^{\prime}$, observing that $g^{*}(z)$ satisfies the hypotheses of $g(z)$ in Theorem of [2].

§ 5. Proof of Theorem 4. Let $\phi\left(z^{2}\right)=f(z) f(-z), g(z)=\phi(-z) / \phi(0)$. If $g(z)$ is constant, then we have $f(z)=A e^{B z}$ where $A, B$ are constants, which is the desired result. Thus we may assume that $g(z)$ is not constant. Since $f(i r) f(-i r) / f(0)^{2}=g\left(r^{2}\right)$, where $r$ is real, we have

$$
\liminf _{r \rightarrow \infty} \frac{\log |g(r)|}{r^{1 / 2}}<\infty
$$


by (1.9). Hence we have by (4.1)

$$
A=\liminf _{r \rightarrow \infty} \frac{\log \left|g^{*}(r)\right|}{r^{1 / 2}}<\infty
$$

where $g^{*}(z)$ is the canonical product with the only negative zeros which have the same absolute values as the zeros of $g(z)$ have. Let

$$
B=\limsup _{r \rightarrow \infty} \frac{\log \left|g^{*}(r)\right|}{r^{1 / 2}} .
$$

If $B$ is $+\infty$ or 0 , then we have a contradiction from $g^{*}(-r)=0(1)$, by the same arguments as those in $\S 3$. If $0<B<+\infty$, then we have $A=B$ and so

$$
n(r, f) \sim \alpha r, \quad \alpha=B / \pi
$$

by Valiron's Tauberian Theorem [4].

We remark that $f(z)=1 / z \Gamma(z)$ satisfies the hypotheses of Theorem 4 .

We can prove Theorem 5 and Theorem $5^{\prime}$ by the similar arguments to those in proof of Theorem 4 and so we omit their proofs.

\section{REFERENCES}

[1] S. Kimura, A characterization of the exponential function by product, Kodai Math. J. 7 (1984), 16-33.

[2] S. Kimura, A characterization of the exponential function by product II, Kodai Math. J. 7 (1984), 317-325.

[3] M. OzAwA, Radial distribution of zeros and deficiency of finite genus, Kodai Math. Sem. Rep. 25 (1973), 502-515.

[4] G. Valiron, Sur les fonctions entières d'ordre fini et d'ordre nul, et en particulier les fonctions à correspondance regulière, Ann. Fac. Sci. Univ. Toulouse (3) 5 (1931), 117-257.

Department of Mathematics

UTSUNOMIYA UNIVERSITY

Mine-Machi, UtSUNOMIYA, JapaN 